\title{
The Existence of Positive Solution of a Nonlinear Problem on Unit Circle
}

\author{
Na Wei, ${ }^{1}$ Yanli Zhou $\mathbb{D}^{2},{ }^{2}$ and Xiangyu Ge $\mathbb{D}^{3}$ \\ ${ }^{1}$ School of Statistics and Mathematics, Zhongnan University of Economics and Law, Wuhan 430073, China \\ ${ }^{2}$ School of Finance, Zhongnan University of Economics and Law, Wuhan 430073, China \\ ${ }^{3}$ Department of Finance, Wuhan Technology and Business University, Wuhan 430065, China
}

Correspondence should be addressed to Yanli Zhou; yanli.zhou1517@zuel.edu.cn

Received 31 May 2020; Accepted 14 July 2020; Published 28 August 2020

Guest Editor: Lishan Liu

Copyright ( $\odot 2020 \mathrm{Na}$ Wei et al. This is an open access article distributed under the Creative Commons Attribution License, which permits unrestricted use, distribution, and reproduction in any medium, provided the original work is properly cited.

We consider the following nonlinear problem $u^{\prime \prime}(x)+\lambda u(x)+f(x, u)=0, \quad x \in S^{1},(\mathrm{P})$ where $S^{1}$ is a unit circle, $\lambda \in \mathbb{R}$ is a parameter, and $f(x, u)$ is a nonlinearity. By using the variational method, we obtain the existence of a positive solution to (P) for all $\lambda \in \mathbb{R}$. This phenomenon is different from the corresponding boundary value problem.

\section{Introduction}

We consider the following nonlinear problem

$$
u^{\prime \prime}(x)+\lambda u(x)+f(x, u)=0, \quad x \in S^{1},
$$

where $S^{1}$ is a unit circle, $\lambda$ is a parameter and the nonlinear term $f$ satisfies.

$\left(f_{1}\right) f(x, t)$ is a continuous function on $S^{1} \times \mathbb{R}$, and $f(x, t)$ is odd on $t$.

The second-order differential equation (1) is a nonlinear elliptic equation model arising from studying some physics processes or geometric problems. For example, (1) is the problem of oscillations $[1,2]$ of a spherical thick shell made of an elastic material when the nonlinearity $f$ satisfies a superlinear condition as $t \longrightarrow+\infty$, namely,

$\left(f_{2}\right) \lim _{t \rightarrow+\infty} f(x, t) / t=+\infty$ uniformly for $x \in S^{1}$.

For $\lambda=1, f(x, t)=g(x)|t|^{p-1} t$ with $p \in \mathbb{R}$ and $g(x)$ : $S^{1} \longrightarrow \mathbb{R},(1)$ is also used to model the planar Minkowski problem [3-5].

As is well known, the strong maximum principle is a powerful tool for studying positive solutions of a nonlinear elliptic equation, see for example $[6,7]$. In $[8]$, the existence of positive solutions to the nonlinear elliptic equation can also be obtained by a standard argument, based on trans- forming the partial differential equation to the equivalent ordinary differential equation. The iterative method can also be used in the existence of a solution to nonlinear elliptic equation (see [9]). If the parameter $\lambda>0$ is small, the related Green function of the operator $d / d x^{2}+\lambda$ on $S^{1}$ is positive. When the Green function is positive, the upper and lower solution method and Schauder's fixed point theorem are also suitable to obtain the positive solution to some nonlinear elliptic equations, see for example [10-12]. However, the Green function of operator $d / d x^{2}+\lambda$ on $S^{1}$ may be signchanging for some $\lambda>0$.

In this paper, we aim at studying the existence of a positive solution of nonlinear equation (1) for all $\lambda \in \mathbb{R}$ and the effect of asymptotic properties of nonlinearity $f(x, t)$ when $t \longrightarrow 0$, to the positivity of solution of (1). Our contribution includes two aspects. Firstly, we establish the following Theorem 1 to show that a nonnegative solution is strictly positive by the analysis based on the Taylor expansion theorem.

Theorem 1. Assume $\lambda \in \mathbb{R}, f$ satisfies $\left(f_{1}\right)$ and $f(x, t) / t$ is bounded as $t \longrightarrow 0$. Let $v \in C^{2}\left(S^{1}\right)$ be a nonnegative solution of (1). If $v \equiv 0$, then $v$ is strictly positive.

Indeed, we can also apply the Green function or the strong maximum principle to study the positive of the solution. However, the direct application of Green function for 
studying the strictly positive of solution often needs more assumptions on parameter $\lambda$ and the information of sign of the non-homogeneous term. By using Theorem 1, we could estimate the strictly positive of solution to (1) for all $\lambda \in \mathbb{R}$ as the following applications.

Secondly, as an application, we give a strictly positive solution of (1) for all $\lambda \in \mathbb{R}$ by using Theorem 1 and the variational method. To study the existence of positive solution to (1) under a general nonlinearity, we also need the following general assumptions.

$\left(f_{3}\right) \lim _{t \rightarrow 0} f(x, t) / t=0$ uniformly for $x \in S^{1}$;

$\left(f_{4}\right)$ there exists $\alpha>2$ such that, for every $x \in S^{1}$ and $t \in \mathbb{R}$

$$
\alpha F(x, t) \leq t f(x, t)
$$

$\left(f_{5}\right)$ there exists $T>0$ such that

$$
\inf _{x \in S^{1},|t|>T} F(x, t)>0
$$

$\left(f_{6}\right)$ for every $x \in S^{1}, f(x, t) / t$ is an increasing function of $t$ on $[0,+\infty)$.

Theorem 2. Assume $\left(f_{1}\right)-\left(f_{6}\right)$ hold. For $\lambda<0$, (1) has a positive solution.

We see that the existence of a positive solution in Theorem 2 depends on the assumption that $\lambda<0$. It is natural to ask whether there exist nontrivial solutions of (1) for some $\lambda \geq 0$. To shed some light to this problem, we give the existence of positive solutions to (1) with some special nonlinearity as follows.

Theorem 3. Assume $f(x, u)=g(x)|u(x)|^{p-1} u(x)$ with $p>1$. Let $\lambda \geq 0$. If $g \in C\left(S^{l}\right)$ is nonnegative with $\int_{S^{1}}|g(x)|^{2 /(1-p)}$ $d x<+\infty$, then (1) has a positive solution.

From Theorems 2 and 3, we obtain that (1) may own a positive solution for all $\lambda \in \mathbb{R}$, which is different from the boundary value problem [13-16]. To illustrate that the conclusions above for (1) is a new phenomenon that different from the existence of a positive solution to boundary value problem, we consider the following problem

$$
\begin{aligned}
& u^{\prime \prime}(x)+\lambda u(x)+g(x)|u(x)|^{p-1} u(x)=0 \quad x \in(0,2 \pi) \\
& u(0)=u(2 \pi)=0
\end{aligned}
$$

Let $u \in C^{2}([0,2 \pi])$ be a solution of $(4)$, we have

$$
\left(\lambda-\frac{1}{4}\right) \int_{0}^{2 \pi} \sin \left(\frac{x}{2}\right) u(x) d x+\int_{0}^{2 \pi} g(x)|u(x)|^{p-1} \sin \left(\frac{x}{2}\right) d x=0 .
$$

If $\lambda>1 / 4$, it follows that there is no positive solution to the boundary value problem (4) with $g(x) \geq 0$.
Since the proof of Theorem 2 depends on $\left(f_{1}\right)$ and $\lambda<0$, it is impossible to deduce Theorem 3 by a similar method for Theorem 2. In the next section, we apply a reverse Hölder's inequality and constrained variational method to prove Theorem 3.

\section{Proof of Theorems}

We first give our new method to estimate the positivity of the solution of (1) as follows. By assumption, we obtain

$$
-v^{\prime \prime}(x)-\lambda v(x)=f(x, v(x)), x \in[0,2 \pi] .
$$

We will prove that $v$ is strictly positive by the method of contradiction. Assume $v\left(\theta_{0}\right)=0$ for some $\theta_{0} \in[0,2 \pi]$. We choose $\theta_{0}=\inf \{x \in[0,2 \pi]: v(x)=0\}$. Since $v \equiv 0$, we have two points $\theta_{r}$ and $\theta_{l}$ in $[0,2 \pi]$ such that $\theta_{0} \leq \theta_{r}<\theta_{l}$ and

$$
v(x)=0 \text { for } x \in\left[\theta_{0}, \theta_{r}\right] \text {; and } v(x)>0 \text { for } x \in\left(\theta_{r}, \theta_{l}\right) .
$$

Since 0 is the minimum value of $v$ on $[0,2 \pi]$, we have

$$
v^{\prime}(x)=0 \text { for } x \in\left[\theta_{0}, \theta_{r}\right]
$$

By $\left(f_{1}\right)$, we see that $f(x, 0)=0$. It follows from (6) and (7) that

$$
v^{\prime \prime}(x)=0 \text { for } x \in\left[\theta_{0}, \theta_{r}\right] .
$$

By Taylor's theorem, we give a formula of $v(x)$ for all $x \in\left(\theta_{r}, \theta_{l}\right)$ as follows,

$$
v(x)=\frac{v^{\prime \prime}\left(\xi_{x}\right)}{2}\left(x-\theta_{r}\right)^{2} \text { for some } \xi_{x} \in\left(\theta_{r}, x\right)
$$

We claim that there exists a strictly monotone decreasing sequence $\left\{\theta_{n}\right\} \subset\left(\theta_{r}, \theta_{l}\right)$ such that

$$
\theta_{n} \stackrel{n}{\longrightarrow} \theta_{r} \text { and }\left|v^{\prime \prime}\left(\theta_{n}\right)\right|>0 .
$$

Otherwise, there exists a small $\delta>0$ such that $\left|v^{\prime \prime}(x)\right|=0$ for all $x \in\left(\theta_{r}, \theta_{r}+\delta\right)$. By applying (10), we derive that

$$
v(x)=0 \text { for } x \in\left(\theta_{r}, \theta_{r}+\delta\right),
$$

which contradicts to the second part of (7). By (9), we see that $v^{\prime \prime}\left(\theta_{r}\right)=0$. Let $\theta_{1}$ be given by $(11)$, then we have

$$
\rho_{1}=\sup \left\{x \in\left[\theta_{r}, \theta_{1}\right]:\left|v^{\prime \prime}(x)\right|=\sup _{\rho \in\left[\theta_{r}, \theta_{1}\right]}\left|v^{\prime \prime}(\rho)\right|\right\}>\theta_{r} .
$$


For $k \in \mathbb{N}$, we can define a sequence of $\rho_{k}$ by

$\rho_{k+1}=\sup \left\{x \in\left(\theta_{r}, \rho_{k}-\delta_{k}\right):\left|v^{\prime \prime}(x)\right|=\sup _{\rho \in\left(\theta_{r}, \rho_{k}-\delta_{k}\right)}\left|v^{\prime \prime}(\rho)\right|\right\}$,

where $\delta_{k}=\left(\rho_{k}-\theta_{r}\right) / 2$. From (11), we see that $\theta_{n} \stackrel{n}{\longrightarrow} \theta_{r}$. Fix $k \in \mathbb{N}$, if $i \in \mathbb{N}$ is large enough, then $\theta_{i} \in\left(\theta_{r}, \rho_{k}\right)$; and it follows from (14) that $\left|v^{\prime \prime}\left(\rho_{k}\right)\right| \geq\left|v^{\prime \prime}\left(\theta_{i}\right)\right|>0$. Following this way, we obtain a strictly monotone decreasing sequence $\left\{\rho_{n}\right\} \subset\left(\theta_{r}, \theta_{l}\right)$ such that $\rho_{n} \stackrel{n}{\longrightarrow} \theta_{r}$ and

$$
\left|v^{\prime \prime}\left(\rho_{n}\right)\right|>0, \quad\left|v^{\prime \prime}(x)\right| \leq\left|v^{\prime \prime}\left(\rho_{n}\right)\right| \text { for } x \in\left(\theta_{r}, \rho_{n}\right) .
$$

For each $n \in \mathbb{N}$, via applying (10) with $x=\rho_{n}$, there exists $\xi_{n} \in\left(\theta_{r}, \rho_{n}\right)$ such that

$$
v\left(\rho_{n}\right)=\frac{v\left(\xi_{n}\right)}{2}\left(\rho_{n}-\theta_{r}\right)^{2} \text { and }\left|v^{\prime \prime}\left(\xi_{n}\right)\right| \leq\left|v^{\prime \prime}\left(\rho_{n}\right)\right|
$$

Let $x=\rho_{n}$ in (6), we have

$$
-v^{\prime \prime}\left(\rho_{n}\right)-\lambda v\left(\rho_{n}\right)=f\left(\rho_{n}, v\left(\rho_{n}\right)\right)
$$

Substituting $v\left(\rho_{n}\right)$ in (17) by using the first part of (16), and then multiplying (17) by $1 / v^{\prime \prime}\left(\rho_{n}\right)$, we deduce that

$$
-1-\lambda \frac{v^{\prime \prime}\left(\xi_{n}\right)}{2 v^{\prime \prime}\left(\rho_{n}\right)}\left(\rho_{n}-\theta_{r}\right)^{2}=\frac{f\left(\rho_{n}, v\left(\rho_{n}\right)\right)}{v\left(\rho_{n}\right)} \frac{v^{\prime \prime}\left(\xi_{n}\right)}{2 v^{\prime \prime}\left(\rho_{n}\right)}\left(\rho_{n}-\theta_{r}\right)^{2} .
$$

By using the second part of (16) and the assumption that $f(\theta, t) / t$ is bounded, we derive that

$$
\begin{aligned}
\frac{f\left(\rho_{n}, v\left(\rho_{n}\right)\right)}{v\left(\rho_{n}\right)} & =O(1) \text { and } \frac{v^{\prime \prime}\left(\xi_{n}\right)}{2 v^{\prime \prime}\left(\rho_{n}\right)}\left(\rho_{n}-\theta_{r}\right)^{2} \\
& =o(1) \text { as } n \longrightarrow+\infty .
\end{aligned}
$$

From (18), we get a contradiction that $1=o(1)$ as $n \longrightarrow+\infty$.

To prove Theorem 2, we firstly obtain a nonnegative solution of (1) via a minimum of $I$ on manifold $N$; then, we prove that it is strictly positive by using Theorem 1 . The process is standard for studying the existence of nonnegative solutions to nonlinear elliptic equations by the method involving the Nehari manifold. For completeness, we give the main steps. The following deformation lemma is needed.
Lemma 1. (see, for example, Lemma 2.3 in [17]). Let $X$ be a Banach space, $I \in C^{l}(X, \mathbb{R}), S \subset X, c \in \mathbb{R}, \varepsilon, \delta>0$ such that

$$
\begin{aligned}
\inf _{\phi=0} \frac{I^{\prime}(u) \phi}{\|\phi\|} & \geq 8 \varepsilon / \delta \text { for all } u \\
& \in\{u:\|u-v\| \leq 2 \delta, c-2 \leq \varepsilon I(u) \leq c+2 \varepsilon\} .
\end{aligned}
$$

We denote by $I^{d}$ the set $\{u \in X: I(u) \leq d\}$ for any $d \in \mathbb{R}$. Then, there exists $\eta \in C([0,1] \times X, X)$ such that

(i) $\eta(t, u)=u$, if $t=0$ or if $u \in\left\{u \in X: \inf _{w \in S}\|u-w\| \leq 2 \delta\right.$, $c-2 \varepsilon \leq I(u) \leq c+2 \varepsilon\}$,

(ii) $\eta\left(1, I^{c+\varepsilon} \cap S\right) \subset I^{c-\varepsilon}$

(iii) $\eta(t, \cdot)$ is an homeomorphism of $X$ for any $t \in[0,1]$

(iv) $I(\eta(\cdot, u))$ is non increasing for any $u \in X$

Let $S$ be parameterized by angle $x, H:=H^{1}(S)$ be the Sobolev space equipped with the usual normal

$$
\|u\|=\left\{\int_{S}\left(u^{\prime}\right)^{2}+u^{2} d x\right\}^{1 / 2}
$$

Define $F(x, u)=\int_{0}^{u} f(x, t) d t$. Under the assumptions $\left(f_{1}\right)-\left(f_{3}\right)$, we have the following $C^{1}$ variational function of (1).

$$
I(u)=\frac{1}{2} \int_{S^{1}} u^{\prime 2} d x-\frac{\lambda}{2} \int_{S^{1}} u^{2} d x-\int_{S^{1}} F(x, u) d x .
$$

The related Nehari manifold is defined by

$$
\begin{aligned}
N:= & \left\{u \in H^{1}\left(S^{1}\right) \backslash\{0\}: \int_{S^{1}} u^{\prime 2} d x-\lambda \int_{S^{1}} u^{2} d x\right. \\
& \left.-\int_{S^{1}} f(x, u) u d x=0\right\} .
\end{aligned}
$$

Similar to Lemma 4.1 in [17], we have the following lemma.

Lemma 2. Assume $\left(f_{1}\right)-\left(f_{6}\right)$ hold. Let $u \in H$ be nontrivial and $h(t)=I(t u)$ for $t>0$. If $\lambda<0$, there exists $t_{u}>0$ such that $t_{u} u \in N, h\left(t_{u}\right)=\max _{t>0} h(t)$. The map $u \longrightarrow t_{u}$ is continuous, and $u \longrightarrow t_{u} u$ defines a homeomorphism of the unit sphere of $H$ with $N$.

Proof. If $\lambda<0$, we see that $1 / 2 \int_{S^{1}} u^{\prime 2} d x-\lambda / 2 \int_{S^{1}} u^{2} d x>0$ for the nontrivial $u \in H$. For any $u \in N$, let $g(t)=1 / t \int_{S^{1}} f(x, t u)$ $d x$. Then $g$ is an increasing function of $t$ by $\left(f_{6}\right)$. By the definition of $h(t)=I(t u)$, we see that $h(0)=0$, and $t_{u} u \in N$ if and only if $\int_{S^{1}} u^{\prime 2} d x-\lambda \int_{S^{1}} u^{2} d x=1 / t_{u} \int_{S^{1}} f\left(x, t_{u} u\right) u d x$, which is equivalent to $h^{\prime}\left(t_{u}\right)=0$. Under the assumptions $\left(f_{3}\right)-\left(f_{5}\right)$, 
we see that $h(t)>0$ for small $t>0$ and $h(t)<0$ for large $t$. Therefore, there exists a unique $t_{u}$ such that $h^{\prime}\left(t_{u}\right)=0$ and $t_{u} u \in N$. By $\left(f_{4}\right)$, there exists a constant $C_{0}>0$ such that

$$
C_{0}\left(|u|^{\alpha}-1\right) \leq u f(x, u)
$$

where $\alpha>2$ is given by $\left(f_{4}\right)$.

Assume that there exists a nontrivial sequence $u_{n} \stackrel{n}{\longrightarrow} u$ in $H^{1}\left(S^{1}\right)$ with $u=0$, we have a sequence $\left\{t_{u_{n}}\right\}$ that $t_{u_{n}} u_{n} \in$ $N$. This and $\left(f_{4}\right)$ imply that

$$
\begin{aligned}
C t_{u_{n}}\left\|u_{n}\right\|^{2} & \geq t_{u_{n}}\left(\int_{S^{1}} u_{n^{\prime}}^{2} d x-\lambda \int_{S^{1}} u_{n}^{2} d x\right) \\
& =\int_{S^{1}} f\left(x, t_{u_{n}} u_{n}\right) u_{n} d x \\
& \geq \int_{S^{1}} C_{0}\left(t_{u_{n}}^{\alpha}\left|u_{n}\right|^{\alpha}-1\right) d x .
\end{aligned}
$$

It follows that $\left\{t_{u_{n}}\right\}$ is bounded. And a subsequence of $\left\{t_{u_{n}}\right\}$ converges to a number $t_{0}$. By using (25) and the uniqueness of map $t_{u}=t(u)$, we see that $t_{0}=t_{u}$. That is, $t_{u_{n}}$ converge to $t_{u}$. The inverse map of $u \longrightarrow t_{u} u$ is the retraction $u \longrightarrow u /\|u\|$.

By using the properties of $I$ in Lemma 2. We obtain the following Lemma 3.

Lemma 3. Assume $\left(f_{1}\right)-\left(f_{6}\right)$ hold and $\lambda<0$. Let $c_{1}=\inf _{u \in N} I(u)$ , then $c_{1}>0$. If $v \in N$ and $I(v)=c_{1}$, then $v$ is a critical point of $I$ on $H$.

Proof. Let $c_{2}:=\inf _{u \in H, u=0} \max _{t \geq 0} I(t u)$ and $c:=\inf _{\gamma \in \Gamma} \max _{t \in[0,1]} I(\gamma(t))$, where $\Gamma:=\{\gamma \in C([0,1], H): I(\gamma(1))<0, \gamma(0)=0\}$. We prove this Lemma by the following two steps.

Step 1. $c_{1}=c_{2}=c>0$.

By Lemma 2, we see that $I\left(t_{u} u\right)=\max _{t \geq 0} I(t u)$ if and only if $t_{u} u \in N$. This and the definitions of $c_{1}, c_{2}$ imply that $c_{1}=c_{2}$. By $\left(f_{1}\right)-\left(f_{2}\right)$, we see that $I(t u)<0$ for $u=0$ and $t>0$ large. Hence, $c \leq c_{2}$. From Lemma 2, we see that the Nehari manifold $N$ separates $H$ into two parts. By assumptions $\left(f_{1}\right)-$ $\left(f_{3}\right)$, we deduce that $\inf _{u \in N}\|u\|>0$ and there exists $r>0$ such that

$$
\min _{\|u\| \leq r} I(u)=0, \quad \inf _{\|u\|=r} I(u)>0 .
$$

It follows that the pass $\gamma \in \Gamma$ has to cross $N$ and $c \geq \inf _{\|u\|=r} I(u)>0$. These facts imply that $c_{1} \leq c$.

Step 2. $v$ is a critical point of $I$.
Assume that $I^{\prime}(v)=0$. Then there exists $\delta>0$ and $\varepsilon>0$ such that

$$
\inf _{\phi=0} \frac{I^{\prime}(u) \phi}{\|\phi\|} \geq \varepsilon \text { for } \quad \text { all } \quad\|u-v\| \leq 3 \delta .
$$

For $\varepsilon:=\{c / 2, \varepsilon \delta / 8\}$ and $S:=B(v, \delta)$, Lemma 1 yields a deformation $\eta$ such that

(i) $\eta(1, u)=u$ if $u \in\{u \in H: c-\varepsilon \leq I(u) \leq c+2 \varepsilon\}$,

(ii) $\eta\left(1, I^{c+\varepsilon} \cap B(v, \delta)\right) \subset I^{c-\varepsilon}$

(iii) $I(\eta(1, u)) \leq I(u), u \in H$

We deduce that $\max _{t>0} I(\eta(1, t v)) \leq c-\varepsilon<c=c_{2}$, which contradicts the definition of $c_{2}$.

By Lemma 3, we see that $c_{1}=\inf _{u \in N} I(u)>0$. There exists a sequence of $\left\{u_{n}\right\} \subset N$ such that $I\left(u_{n}\right) \longrightarrow c_{1}$ as $n \longrightarrow+\infty$, that is,

$\frac{1}{2} \int_{S^{1}} u^{\prime 2} d x-\frac{\lambda}{2} \int_{S^{1}} u^{2} d x-\int_{S^{1}} F(x, u) d x=c_{1}+o(1)$, as $n \longrightarrow+\infty$,

$\int_{S^{1}} u^{\prime 2} d x-\lambda \int_{S^{1}} u^{2} d x-\int_{S^{1}} f(x, u) u d x=0$,

Let $\alpha>2$ be given by $\left(f_{4}\right)$, it follows from (28) and (29) that

$$
\frac{\alpha-2}{2}\left(\int_{S^{1}} u^{\prime 2} d x-\lambda \int_{S^{1}} u^{2} d x\right) \leq 2 c_{1} \text { for large } n
$$

Since $\lambda<0$, we have that $\left\{\left\|u_{n}\right\|\right\}$ is bounded. Then, there exists a weak limitation $v$ of the sequence $\left\{u_{n}\right\}$ in $H$, i.e., up to a subsequence, $u_{n} \rightarrow v$ weakly in $H$ and $u_{n} \longrightarrow v$ uniformly on $S^{1}$ as $n \longrightarrow+\infty$. This together with the assumptions $\left(f_{1}\right)-\left(f_{3}\right)$ imply that

$$
\lim _{n \rightarrow+\infty} \int_{S^{1}} f\left(x, u_{n}\right) u_{n} d x=\int_{S^{1}} f(x, v) v d x
$$

It follows from (29) and (31) that $\lim _{n \rightarrow+\infty}\left\|u_{n}-v\right\|=0$, which implies that $v \in N$ and $c_{1}=\inf _{u \in N} I(u)=I(v)$. By Lemma 3 , we see that $I^{\prime}(v)=0$. Let $v^{+}=\max \{0, v\}$ and $v^{-}=-\max$ $\{0,-v\}$. Then, we deduce that $I\left(v^{+}\right) v^{+}=I(v) v^{+}=I\left(v^{-}\right) v^{-}=$ $I(v) v^{-}=0$. If $v$ is a sign-changing function, then $v^{-}$and $v^{+}$ are nontrivial. It follows that $v^{-}, v^{+} \in N$. We thus deduce the following contradiction

$$
c_{1}=I(v)=I\left(v^{+}\right)+I\left(v^{-}\right) \geq 2 \inf _{u \in N} I(u)=2 c_{1}>0 .
$$

So, $v$ is not a sign-changing function. By the second part of $\left(f_{1}\right)$, we see that $I(v)=I(-v)$. Without loss of generality, we assume that $v$ is nonnegative. A standard regularity 
shows that $v$ is $C^{2}$ on $S^{1}$. Since the nonlinear term $f$ satisfies assumptions $\left(f_{1}\right)$ and $\left(f_{3}\right)$, we can apply Theorem 1 to get that $v$ is strictly positive.

Let us consider the constrained optimal problem

$$
c=\inf _{F(u)=1} I(u)
$$

where

$$
\begin{aligned}
& I(u)=\int_{S}\left(u^{\prime}\right)^{2}-\lambda u^{2} d x, \\
& F(u)=\int_{S} g(x)|u|^{p+1} d x .
\end{aligned}
$$

For $\lambda \geq 0$, it follows from reverse Hölder inequality and (34) that

$$
\begin{aligned}
\inf _{F(u)=1} I(u) & =\inf _{F(u)=0} \frac{I(u)}{\{F(u)\}^{2 / p+1}} \\
& \geq \inf _{F(u)=0} \frac{-\lambda \int_{S^{1}}|u|^{2} d x}{\left\{\int_{S^{1}} g(x)^{2 /(1-p)} d \theta\right\}^{(1-p) / 2} \int_{S^{1}}|u|^{2} d x} \\
& =-\lambda\left\{\int_{S^{1}} g(x)^{2 /(1-p)} d \theta\right\}^{(p-1) / 2}>-\infty,
\end{aligned}
$$

which implies that $I(u)$ is bounded in $H^{1}(S)$ from below under the constrained condition that $F(u)=1$. By the definition of $I, F$ in (34) and (35), there exists a nonnegative minimizing sequence $\left\{u_{n}\right\} \subset H^{1}(S)$ such that $F\left(u_{n}\right)=1$ and

$$
I\left(u_{n}\right) \longrightarrow \inf _{F(u)=1} I(u) \text { as } n \longrightarrow+\infty .
$$

It follows from $2 /(p-1)>(p-1) /(p+1)$ and the Hölder inequality that

$$
\int_{S^{1}} g(x)^{-(p-1) /(p+1)} d x \leq C\left|\int_{S^{1}} g(x)^{2 /(1-p)} d x\right|^{\frac{(p-1)^{2}}{2(p+1)}}<+\infty .
$$

Since $F\left(u_{n}\right)=1$, by Hölder inequality we see that

$$
\begin{aligned}
\int_{S^{1}} u_{n}^{2} d x & \leq\left(\int_{S^{1}} g(x)^{-(p-1) /(p+1)} d x\right)^{(p+1) /(p+2)}\left(\int_{S^{1}} g(x) u_{n}^{p+1} d x\right)^{2 /(p+1)} \\
& =\left(\int_{S^{1}} g(x)^{-(p-1) /(p+1)} d x\right)^{(p+1) /(p+2)}<+\infty
\end{aligned}
$$

This together with $I\left(u_{n}\right) \stackrel{(n \rightarrow+\infty)}{\longrightarrow} \inf _{F(u)=1} I(u)$ imply that $\left\{u_{n}\right\}$ is bounded in $H^{1}(S)$. So, there is a subsequence such that

$$
u_{n} \stackrel{n}{\longrightarrow} v \text { weakly in } H^{1}\left(S^{1}\right) .
$$

By the compactness of Sobolev imbedding, up to a subsequence, we have

$u_{n} \stackrel{n}{\longrightarrow} v$ strongly in $C^{\alpha}\left(S^{1}\right)$ for $\alpha \in(0,1 / 2)$.

It follows that $F(|v|)=1$, and therefore $v=0$.

$$
\int_{0}^{2 \pi} g(\theta)|v|^{p+1} d \theta=\lim _{n \rightarrow+\infty} \int_{0}^{2 \pi} g(\theta)\left|u_{n}\right|^{p+1} d \theta=1
$$

By the Fatou lemma, we get

$$
\inf _{F(u)=1} I(u) \leq I(|v|) \leq \lim _{n \rightarrow+\infty} I\left(u_{n}\right)=\inf _{F(u)=1} I(u)
$$

So, $|v|$ is a minimizer of $I$ under constrained condition that $F(u)=1$. There exists $\omega \in \mathbb{R}$ such that

$$
I^{\prime}(|v|)=\omega F^{\prime}(|v|) \text { in } H^{-1}(S) .
$$

It follows from the regularity theorem that $|v| \in C^{2}(S)$ is a classical nonnegative solution of

$$
-u^{\prime \prime}-\lambda u=\frac{\omega(p+1)}{2} g(x)|u|^{p-1} u, x \in S^{1} .
$$

Let $w=(\omega(p+1) / 2)^{1 /(p-1)}|v|$, we see that $w$ is nonnegative solution of (1) with $f(x, u)=g(x)|u|^{p-1} u$. By applying Theorem 1 , we see that $w$ is positive.

\section{Data Availability}

No data were used to support this study.

\section{Conflicts of Interest}

The authors declare that there is no conflict of interests regarding the publication of this paper.

\section{Acknowledgments}

This work was supported by the National Natural Science Foundation of China (NSFC) (Nos. 71901222 and 71974204), the Natural Science Foundation of Hubei Province (No. 2019CFB570), and "the Fundamental Research Funds for the Central Universities", Zhongnan University of Economics and Law (Nos. 2722019PY053 and 2722020JCT008).

\section{References}

[1] M. del Pino and R. F. Manásevich, "Infinitely many Tperiodic solutions for a problem arising in nonlinear 
elasticity," Journal of Differential Equations, vol. 103, pp. 260-277, 1993.

[2] Z. Guo and R. Solecki, "Free and forced finite amplitude oscillations of an elastic thick-walled hollow sphere made of incompressible material," Archiwum Mechaniki Stosowanej, vol. 15, pp. 427-433, 1963.

[3] J. Dou and M. Zhu, "The two dimensional Lp Minkowski problem and nonlinear equations with negative exponents," Advances in Mathematics, vol. 230, no. 3, pp. 1209-1221, 2012.

[4] Y. Jiang and Y. Wu, "On the 2-dimensional dual Minkowski problem," Journal of Differential Equations, vol. 263, no. 6, pp. 3230-3243, 2017.

[5] N. Wei, "The solvability of dual Minkowski problem in $\mathbb{R}^{2}$," Acta Mathematica Scientia, Series A, Chinese Edition, vol. 39, no. 6, pp. 1314-1322, 2019.

[6] H. Zhou, "Positive solutions for a Dirichlet problem," Acta Mathematicae Applicatae Sinica, vol. 17, no. 3, pp. 340-349, 2001.

[7] H. Zhou, "Positive solutions for nonhomogeneous elliptic equations with critical growth on $\mathbb{R}^{2}$," Acta Mathematica Scientia, vol. 18, no. 2, pp. 124-138, 1998.

[8] X. Zhang, J. Jiang, Y. Wu, and Y. Cui, "Existence and asymptotic properties of solutions for a nonlinear Schrödinger elliptic equation from geophysical fluid flows," Applied Mathematics Letters, vol. 90, pp. 229-237, 2019.

[9] X. Zhang, J. Jiang, Y. Wu, and Y. Cui, “The existence and nonexistence of entire large solutions for a quasilinear Schrödinger elliptic system by dual approach," Applied Mathematics Letters, vol. 100, article 106018, 2020.

[10] X. Hao, M. Zuo, and L. Liu, "Multiple positive solutions for a system of impulsive integral boundary value problems with sign-changing nonlinearities," Applied Mathematics Letters, vol. 82, pp. 24-31, 2018.

[11] J. He, X. Zhang, L. Liu, Y. Wu, and Y. Cui, "Existence and asymptotic analysis of positive solutions for a singular fractional differential equation with nonlocal boundary conditions," Boundary Value Problems, vol. 2018, no. 1, Article ID 189, 2018.

[12] J. Jiang, L. Liu, and Y. Wu, "Second-order nonlinear singular Sturm-Liouville problems with integral boundary conditions," Applied Mathematics and Computation, vol. 215, no. 4, pp. 1573-1582, 2009.

[13] J. He, X. Zhang, L. Liu, and Y. Wu, "Existence and nonexistence of radial solutions of the Dirichlet problem for a class of general $k$-Hessian equations," Nonlinear Analysis: Modelling and Control, vol. 23, pp. 475-492, 2018.

[14] X. Zhang, L. Liu, and Y. Wu, "Multiple positive solutions of a singular fractional differential equation with negatively perturbed term," Mathematical and Computer Modelling, vol. 55, no. 3-4, pp. 1263-1274, 2012.

[15] X. Zhang, L. Liu, Y. Wu, and Y. Cui, "A sufficient and necessary condition of existence of blow-up radial solutions for a $k$-Hessian equation with a nonlinear operator," Nonlinear Analysis: Modelling and Control, vol. 25, no. 1, pp. 126-143, 2020.

[16] X. Zhang, Y. Wu, and L. Caccetta, "Nonlocal fractional order differential equations with changing-sign singular perturbation," Applied Mathematical Modelling, vol. 39, no. 21, pp. 6543-6552, 2015.

[17] M. Willem, Minimax Theorems, Birkhauser, Basel, 1996. 